\title{
Identifying Higher-Volume Antibiotic Outpatient Prescribers Using Publicly Available Medicare Part D Data — United States, 2019
}

\author{
Katryna A. Gouin, $\mathrm{MPH}^{1}$; Katherine E. Fleming-Dutra, $\mathrm{MD}^{2}$; Sharon Tsay, MD²; Destani Bizune, $\mathrm{MPH}^{2}$; Lauri A. Hicks, DO²; Sarah Kabbani, MD²
}

Antibiotic prescribing can lead to adverse drug events and antibiotic resistance, which pose ongoing urgent public health threats (1). Adults aged $\geq 65$ years (older adults) are recipients of the highest rates of outpatient antibiotic prescribing and are at increased risk for antibiotic-related adverse events, including Clostridioides difficile and antibiotic-resistant infections and related deaths (1). Variation in antibiotic prescribing quality is primarily driven by prescribing patterns of individual health care providers, independent of patients' underlying comorbidities and diagnoses (2). Engaging higher-volume prescribers (the top $10 \%$ of prescribers by antibiotic volume) in antibiotic stewardship interventions, such as peer comparison audit and feedback in which health care providers receive data on their prescribing performance compared with that of other health care providers, has been effective in reducing antibiotic prescribing in outpatient settings and can be implemented on a large scale (3-5). This study analyzed data from the Centers for Medicare \& Medicaid Services (CMS) Part D Prescriber Public Use Files (PUFs)* to describe higher-volume antibiotic prescribers in outpatient settings compared with lower-volume prescribers (the lower $90 \%$ of prescribers by antibiotic volume). Among the 59.4 million antibiotic prescriptions during $2019,41 \%$ (24.4 million) were prescribed by the top $10 \%$ of prescribers $(69,835)$. The antibiotic prescribing rate of these higher-volume prescribers ( 680 prescriptions per 1,000 beneficiaries) was $60 \%$ higher than that of lower-volume prescribers (426 prescriptions per 1,000 beneficiaries). Identifying health care providers responsible for a higher volume of antibiotic prescribing could provide a basis for additional assessment of appropriateness and outreach. Public health organizations and health care systems can use publicly available data to guide focused interventions to optimize antibiotic prescribing to limit the emergence of antibiotic resistance and improve patient outcomes.

Approximately $70 \%$ of Medicare beneficiaries are enrolled in Medicare Part D, the prescription drug benefit program for adults aged $\geq 65$ years and persons with disabilities or end-stage renal disease. CMS Medicare Part D Prescribers by Provider is a publicly available data set that contains prescriber-level aggregate counts of outpatient prescription drug events by three drug types (antibiotics, antipsychotics, and opioids) and

\footnotetext{
*https://data.cms.gov/provider-summary-by-type-of-service/medicare-part-dprescribers/medicare-part-d-prescribers-by-provider (Accessed October 18, 2021).
}

provider characteristics, including names, National Provider Identifier, specialty (including prescriber type), and zip code. There is a 2-year lag in data availability, during which prescription drug claims are finalized. Because beneficiary and antibiotic claim counts fewer than 11 are suppressed, the 2019 Medicare Part D Prescribers by Provider data set was used to assess prescriber-level antibiotic prescriptions among health care providers in the United States who distributed 11 or more antibiotic prescriptions.

Higher-volume prescribers were defined as those in the highest 10th percentile of prescriber-level antibiotic volume (number of antibiotic prescriptions filled) across all Medicare providers nationwide. The cumulative percentage of antibiotic volume prescribed by higher-volume prescribers was assessed overall, and the percentage of higher-volume prescribers in each U.S. Census Bureau region ${ }^{\dagger}$ and specialty were described. To verify that antibiotic volume was not exclusively driven by the number of Medicare beneficiaries attributed to an individual prescriber, the percentage of beneficiaries with an antibiotic prescription and the prescriber's antibiotic volume per 1,000 beneficiaries were calculated. The antibiotic prescribing rate was compared between the defined national subset of higher-volume prescribers and lower-volume prescribers by specialty and U.S. Census Bureau region. Ten beneficiaries were imputed for suppressed beneficiary counts to provide a conservative estimate of the prescribing rate. The Wilcoxon rank-sum test was used to compare median prescribing rates among prescribers. All analyses were performed using SAS (version 9.4; SAS Institute). This activity was reviewed by CDC and was conducted consistent with applicable federal law and CDC policy. $\$$

During 2019, the Medicare Part D Prescribers by Provider data set included 1.2 million prescribers. After excluding prescribers with fewer than 11 antibiotic prescriptions and

\footnotetext{
$\dagger$ U.S. Census Bureau regions: Northeast: Connecticut, Maine, Massachusetts, New Hampshire, New Jersey, New York, Pennsylvania, Rhode Island, and Vermont. Midwest: Illinois, Indiana, Iowa, Kansas, Michigan, Minnesota, Missouri, Nebraska, North Dakota, Ohio, South Dakota, and Wisconsin. South: Alabama, Arkansas, Delaware, District of Columbia, Florida, Georgia, Kentucky, Louisiana, Maryland, Mississippi, North Carolina, Oklahoma, South Carolina, Tennessee, Texas, Virginia, and West Virginia. West: Alaska, Arizona, California, Colorado, Hawaii, Idaho, Montana, Nevada, New Mexico, Oregon, Utah, Washington, and Wyoming.

$\$ 45$ C.F.R. part 46.102(l)(2), 21 C.F.R. part 56; 42 U.S.C. Sect. 241 (d); 5 U.S.C. Sect. 552a; 44 U.S.C. Sect. 3501 et seq.
} 
those in U.S. territories or overseas military bases, 697,065 $(56 \%)$ prescribers were included in the analysis. A total of 59.4 million antibiotic prescriptions were filled by Part D beneficiaries, with a median of $47(\mathrm{IQR}=23-100)$ antibiotic prescriptions per prescriber. Among all antibiotic prescriptions, $41 \%$ (24.4 million) were written by the top $10 \%(69,835)$ of antibiotic prescribers by number of prescriptions written (antibiotic volume) (Figure); these prescribers wrote a median of 284 antibiotic prescriptions $(\mathrm{IQR}=230-393)$ compared with a median of 41 (IQR = 21-78) among lower-volume prescribers. Higher-volume prescribers prescribed antibiotics to a median of $38 \%$ of their patient panel (i.e., group of patients assigned to a specific health care provider or clinical team) compared with a median of $32 \%$ among lower-volume prescribers. In addition, the median antibiotic prescribing rate among higher-volume prescribers was $60 \%$ higher than that of lower-volume prescribers (680 versus 426 prescriptions per 1,000 beneficiaries) $(\mathrm{p}<0.001)$.

Approximately one half (48\%) of higher-volume prescribers practiced in the South and prescribed 49\% (12.3 million) of the total antibiotic prescriptions in this region (Table). Higher-volume prescribers in the South also had the highest median antibiotic prescribing rate (696 antibiotic prescriptions per 1,000 beneficiaries) compared with higher-volume prescribers in other regions (649 in the West) $(\mathrm{p}<0.001)$. The most common specialties of higher-volume prescribers were family practice and internal medicine, with $21 \%(19,213$ of $89,759)$ and $20 \%(17,185$ of 85,442$)$ of prescribers, respectively classified as higher-volume prescribers. Family practice and internal medicine higher-volume prescribers accounted for approximately $60 \%$ of the antibiotics prescribed within their respective specialties and $22 \%$ of the total antibiotic volume, collectively. Although urologists only contributed $1 \%$ of the total prescriber number during 2019 , one half $(50 \%)$ of urologists were higher-volume prescribers and prescribed 2.0 million antibiotic prescriptions, or $83 \%$ of urology-prescribed antibiotic volume. Higher-volume prescribers, as expected, had higher antibiotic prescribing rates within each specialty, with the highest rate among dentists.

\section{Discussion}

The goal of antibiotic stewardship is to improve the way health care providers prescribe antibiotics to optimize patient outcomes and reduce emergence of antibiotic resistance. During 2019, 41\% of all Medicare Part D antibiotic prescriptions were prescribed by $10 \%$ of antibiotic prescribers, indicating that a small proportion of prescribers accounted for a disproportionately large number of antibiotic prescriptions. A similar 2016 study using claims data in Tennessee found that $50 \%$ of the state's antibiotic volume was attributed to $9 \%$ of prescribers (6). This substantial difference in prescribing practices presents opportunities for improved prescribing through antibiotic

FIGURE. Cumulative percentage of antibiotics prescribed by Medicare Part $\mathrm{D}^{*}$ prescribers, by prescribing volume and rate among highervolume and lower-volume prescribers ${ }^{\dagger}$ — United States, 2019

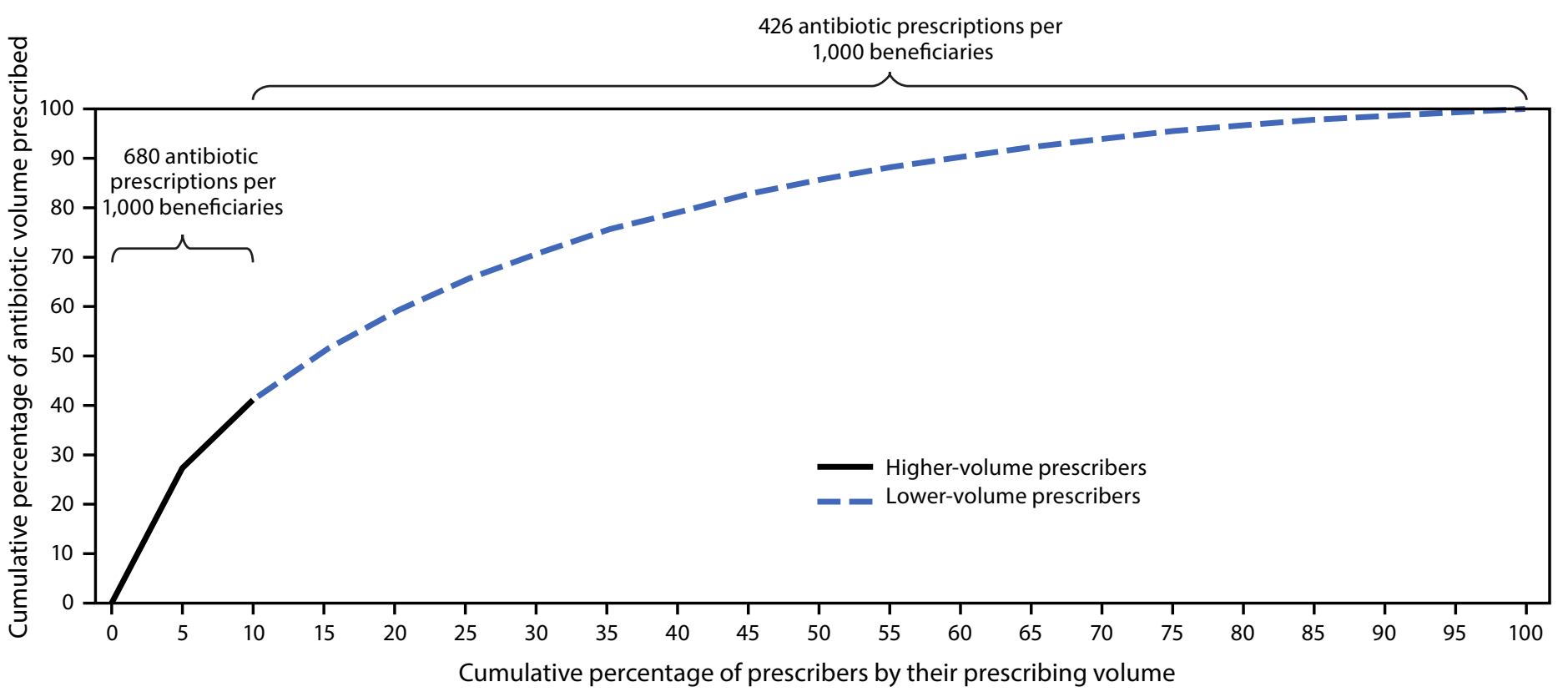

* Centers for Medicare \& Medicaid Services Part D Prescribers by Provider data set, 2019.

${ }^{\dagger}$ Higher-volume prescribers are the top $10 \%$ of prescribers by antibiotic volume; lower-volume prescribers are the lower $90 \%$ of prescribers by antibiotic volume. 
TABLE. Number of antibiotic prescribers, number of outpatient antibiotic prescriptions, and prescribing rate per 1,000 beneficiaries* among higher-volume prescribers and lower-volume prescribers, ${ }^{\dagger}$ by U.S. Census Bureau region and specialty - United States, 2019

\begin{tabular}{|c|c|c|c|c|c|c|c|c|c|}
\hline \multirow[b]{3}{*}{ Characteristics } & \multicolumn{3}{|c|}{ Higher-volume prescribers (top 10\%) } & \multicolumn{3}{|c|}{ Lower-volume prescribers (lower $90 \%$ ) } & \multicolumn{3}{|c|}{ Total prescribers $\S$} \\
\hline & $\begin{array}{l}\text { Prescribers } \\
(n=69,835)\end{array}$ & $\begin{array}{c}\text { Prescriptions } \\
\text { ( } n=24.4 \text { million })\end{array}$ & $\begin{array}{l}\text { Prescriptions per } \\
1,000 \text { beneficiaries }\end{array}$ & $\begin{array}{l}\text { Prescribers } \\
(n=627,230)\end{array}$ & $\begin{array}{c}\text { Prescriptions } \\
(\mathrm{n}=35.0 \text { million })\end{array}$ & $\begin{array}{l}\text { Prescriptions per } \\
1,000 \text { beneficiaries }\end{array}$ & $\begin{array}{l}\text { Prescribers } \\
(N=697,065)\end{array}$ & $\begin{array}{c}\text { Prescriptions } \\
(\mathrm{N}=59.4 \text { million })\end{array}$ & $\begin{array}{l}\text { Prescriptions per } \\
1,000 \text { beneficiaries }\end{array}$ \\
\hline & No. (\%) & No. (\%) & Median (IQR) & No. (\%) & No. (\%) & Median (IQR) & No. (\%) & No. (\%) & Median (IQR) \\
\hline \multicolumn{10}{|c|}{ U.S. Census Bureau region? } \\
\hline South & $33,571(48.1)$ & $12,277,664(50.3)$ & $696(516-925)$ & $217,854(34.7)$ & $12,800,940(36.6)$ & $434(250-714)$ & $251,425(36.1)$ & $25,078,604(42.2)$ & $471(277-765)$ \\
\hline Midwest & $15,096(21.6)$ & $5,163,003(21.2)$ & $681(507-912)$ & $141,561(22.6)$ & $8,110,378(23.2)$ & $435(260-714)$ & $156,657(22.5)$ & $13,273,381(22.4)$ & $461(278-750)$ \\
\hline Northeast & $11,188(16.0)$ & $3,715,665(15.2)$ & $655(472-893)$ & $129,416(20.6)$ & $6,802,148(19.4)$ & $410(224-708)$ & $140,604(20.2)$ & $10,517,813(17.7)$ & $432(238-736)$ \\
\hline West & $9,980(14.3)$ & $3,241,995(13.3)$ & $649(467-879)$ & $138,399(22.1)$ & $7,270,835(20.8)$ & $419(230-731)$ & $148,379(21.3)$ & $10,512,830(17.7)$ & $436(240-750)$ \\
\hline \multicolumn{10}{|l|}{ Specialty } \\
\hline Family practice & $19,213(27.5)$ & $6,815,010(27.9)$ & $611(463-796)$ & $70,546(11.2)$ & $5,341,667(15.3)$ & $303(201-455)$ & $89,759(12.9)$ & $12,156,677(20.5)$ & $358(225-553)$ \\
\hline $\begin{array}{l}\text { Internal } \\
\text { medicine }\end{array}$ & $17,185(24.6)$ & $6,476,428(26.5)$ & $590(429-816)$ & $68,257(10.9)$ & $4,716,606(13.5)$ & 333 (209-477) & $85,442(12.3)$ & $11,193,034(18.8)$ & $375(237-545)$ \\
\hline $\begin{array}{l}\text { Nurse } \\
\text { practitioner }\end{array}$ & $9,857(14.1)$ & $2,920,894(12.0)$ & $711(553-866)$ & $98,182(15.7)$ & $5,934,913(17.0)$ & $398(244-587)$ & $108,039(15.5)$ & $8,855,807(14.9)$ & $425(258-625)$ \\
\hline Urology & $4,738(6.8)$ & $2,020,285(8.3)$ & $760(603-961)$ & 4,687 (0.7) & $426,424(1.2)$ & $500(370-660)$ & $9,425(1.4)$ & $2,446,709(4.1)$ & $632(462-839)$ \\
\hline $\begin{array}{l}\text { Physician } \\
\text { assistant }\end{array}$ & $5,200(7.4)$ & $1,553,698(6.4)$ & $686(537-816)$ & $61,273(9.8)$ & $3,634,949(10.4)$ & $407(251-567)$ & $66,473(9.5)$ & $5,188,647(8.7)$ & $427(265-594)$ \\
\hline Dentist & $2,063(3.0)$ & $552,858(2.3)$ & $1,271(1,122-1,450)$ & $110,629(17.6)$ & $5,004,506(14.3)$ & $1,068(914-1,222)$ & $112,692(16.2)$ & $5,557,364(9.4)$ & $1,071(917-1,228)$ \\
\hline Other** & $11,579(16.6)$ & $4,059,154(16.6)$ & $850(583-1,239)$ & $213,656(34.1)$ & $9,925,236(28.4)$ & $360(188-533)$ & $225,235(32.3)$ & $13,984,390(23.5)$ & $375(197-560)$ \\
\hline
\end{tabular}

Abbreviation: $\mathrm{CMS}=$ Centers for Medicare \& Medicaid Services.

* CMS Part D Prescribers by Provider data set, 2019.

† Higher-volume prescribers are the top $10 \%$ of prescribers by antibiotic volume; lower-volume prescribers are the lower $90 \%$ of prescribers by antibiotic volume.

$\S$ Total number of prescribers includes prescribers with $\geq 11$ antibiotic prescription drug events filled at their direction by Medicare Part D beneficiaries during 2019.

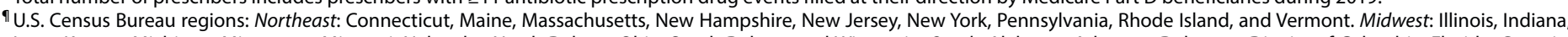

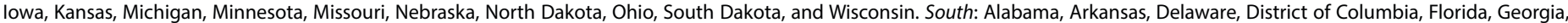

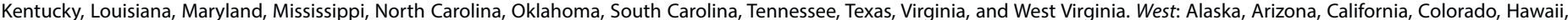
Idaho, Montana, Nevada, New Mexico, Oregon, Utah, Washington, and Wyoming.

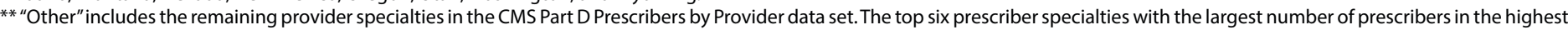
10th percentile by antibiotic prescription volume are represented.

stewardship activities focusing on these higher-volume prescribers, independent of specialty. Total antibiotic volume is associated with unnecessary prescribing rates and might be a reasonable proxy for unnecessary prescribing in primary care settings ( 7 ). Furthermore, higher-volume prescribers prescribed antibiotics to a larger share of their patient panel and their prescribing rate was $60 \%$ higher than that of lower-volume prescribers, indicating that their prescribing practices might be independent of the number of beneficiaries under their care. Thus, prioritizing higher-volume prescribers for focused stewardship interventions has the potential to have a sizeable impact on antibiotic prescribing, even when data on visit volume, prescribing indications, and appropriateness are not available.

This study demonstrates a way to identify antibiotic prescribers who account for a large proportion of prescribing and could provide a basis for additional assessment of appropriateness and outreach. For example, public health organizations could use Medicare Part D data to identify individual higher-volume antibiotic prescribers by specialty for focused stewardship interventions. The higher-volume prescribers in primary care specialties prescribed one-quarter of the total Medicare Part D antibiotic volume during 2019. Studies indicate that primary care providers have varying prescribing rates, suggesting opportunities for improvement in settings in which most antibiotics are prescribed (5). Urologists and dentists also have high prescribing rates and should be considered for antibiotic stewardship interventions $(8,9)$. Further evaluation of prescribing practices by and within specialties and specific conditions are needed to identify areas for improvement in antibiotic prescribing. Similar to this analysis, studies have described higher rates of total outpatient antibiotic prescribing in the South (8), which could not be explained by differences in underlying conditions in older adults (10). Further evaluation of inequities in social determinants of health, underlying patient comorbidities, and access to care is needed to assess whether these factors might contribute to higher rates of prescribing observed in the South.

The publicly available CMS Part D Prescribers by Provider data set might enable public health organizations and health care systems to efficiently identify prescribers for stewardship outreach in their jurisdictions without the need for complex analytic methods or need to acquire prescription claims data or diagnosis data. Prioritizing higher-volume prescribers for antibiotic stewardship interventions could facilitate larger reductions than targeting lower-volume prescribers. Prescriber feedback letters with peer comparison, which is an evidencebased, low-cost, and scalable intervention (3-5) can be used to engage specific health care providers or geographic areas. ${ }^{* *}$ In

\footnotetext{
Ittps://www.cdc.gov/antibiotic-use/pdfs/Outpatient-Rx-Analytic-Guide-508.pdf ** CDC provides outpatient antibiotic stewardship resources, including prescriber feedback letters. https://www.cdc.gov/antibiotic-use/core-elements/outpatient.html
} 


\section{Summary}

What is already known about this topic?

Health care providers vary in their propensity to prescribe antibiotics. Peer comparison audit and feedback is an effective antibiotic stewardship intervention to improve antibiotic prescribing.

What is added by this report?

The highest $10 \%$ of antibiotic prescribers prescribed $41 \%$ of total antibiotic prescriptions for Medicare Part D beneficiaries in 2019. The antibiotic prescribing rate of these higher-volume prescribers was $60 \%$ higher than that of lower-volume prescribers.

What are the implications for public health practice?

Publicly available Medicare Part D data can be used by public health organizations and health care systems to guide antibiotic stewardship interventions and optimize antibiotic prescribing to limit the emergence of antibiotic resistance and improve patient outcomes.

a randomized clinical trial among primary care physicians in Ontario, Canada receipt of a single letter informing prescribers they were in the top 25th percentile of prescribed antibiotic volume compared with their peers, along with recommendations about prescribing duration, resulted in a $5 \%$ relative reduction in total antibiotic use (4).

The findings in this report are subject to at least four limitations. First, the CMS Part D Prescribers by Provider data set captured prescription claims submitted to Medicare Part D and is thus not representative of the entire older adult population. Second, these data might not reflect health care providers' prescribing behavior for their entire patient population and might overrepresent health care providers with a larger share of Medicare beneficiaries, patients with complex medical conditions, or visits for conditions for which antibiotics are prescribed. Third, this data only describes volume of prescribing and does not report diagnosis and underlying conditions; therefore, the data cannot be used to assess appropriateness of prescribing. Finally, the 2-year lag in data availability affects timeliness, which would be important for real-time audit and feedback. Nonetheless, these data are useful for characterizing provider prescribing behaviors and supporting public health stewardship outreach.

This report demonstrates how publicly available data might be leveraged to monitor antibiotic use and identify highervolume prescribers. CMS Part D Prescribers by Provider data can be used by public health organizations and health care systems to guide antibiotic stewardship interventions and optimize antibiotic prescribing to limit the emergence of antibiotic resistance and improve patient outcomes.
Corresponding author: Katryna A. Gouin, kgouin@cdc.gov, 860-810-8061.

${ }^{1}$ Chenega Corporation, Anchorage, Alaska; ${ }^{2}$ Division of Healthcare Quality Promotion, National Center for Emerging and Zoonotic Infectious Diseases, CDC.

All authors have completed and submitted the International Committee of Medical Journal Editors form for disclosure of potential conflicts of interest. Lauri A. Hicks reports being an unpaid elected board member of the Society for Healthcare Epidemiology of America and an unpaid member of the American College of Physicians Clinical Guidelines Committee. No other potential conflicts of interest were disclosed.

\section{References}

1. CDC. Antibiotic resistance threats in the United States, 2019. Atlanta, GA: US Department of Health and Human Services, CDC; 2019. https://www.cdc.gov/drugresistance/pdf/threats-report/2019-ar-threatsreport-508.pdf

2. Jones BE, Sauer B, Jones MM, et al. Variation in outpatient antibiotic prescribing for acute respiratory infections in the veteran population: a cross-sectional study. Ann Intern Med 2015;163:73-80. PMID:26192562 https://doi.org/10.7326/M14-1933

3. King LM, Fleming-Dutra KE, Hicks LA. Advances in optimizing the prescription of antibiotics in outpatient settings. BMJ 2018;363:k3047. PMID:30420401 https://doi.org/10.1136/bmj.k3047

4. Schwartz KL, Ivers N, Langford BJ, et al. Effect of antibiotic-prescribing feedback to high-volume primary care physicians on number of antibiotic prescriptions: a randomized clinical trial. JAMA Intern Med 2021;181:1165-73. PMID:34228086 https://doi.org/10.1001/ jamainternmed.2021.2790

5. Hallsworth M, Chadborn T, Sallis A, et al. Provision of social norm feedback to high prescribers of antibiotics in general practice: a pragmatic national randomised controlled trial. Lancet 2016;387:1743-52. PMID:26898856 https://doi.org/10.1016/S0140-6736(16)00215-4

6. Staub MB, Ouedraogo Y, Evans CD, et al. Analysis of a high-prescribing state's 2016 outpatient antibiotic prescriptions: implications for outpatient antimicrobial stewardship interventions. Infect Control Hosp Epidemiol 2020;41:135-42. PMID:31755401 https://doi.org/10.1017/ ice. 2019.315

7. Kitano T, Langford BJ, Brown KA, et al. The association between high and unnecessary antibiotic prescribing: a cohort study using family physician electronic medical records. Clin Infect Dis 2021;72:e345-51. PMID:32785696 https://doi.org/10.1093/cid/ciaa1 139

8. Hicks LA, Bartoces MG, Roberts RM, et al. US outpatient antibiotic prescribing variation according to geography, patient population, and provider specialty in 2011. Clin Infect Dis 2015;60:1308-16. PMID:25747410 https://doi.org/10.1093/cid/civ076

9. Kabbani S, Palms D, Bartoces M, Stone N, Hicks LA. Outpatient antibiotic prescribing for older adults in the United States: 2011 to 2014. J Am Geriatr Soc 2018;66:1998-2002. PMID:30221746 https://doi. org/10.1111/jgs.15518

10. Zhang Y, Steinman MA, Kaplan CM. Geographic variation in outpatient antibiotic prescribing among older adults. Arch Intern Med 2012;172:1465-71. PMID:23007171 https://doi.org/10.1001/ archinternmed.2012.3717 